\title{
Programa para facilitar la detección precoz de trastornos mentales graves en el medio escolar: lecciones aprendidas de una experiencia piloto.
}

A program to provide early detection of mental disorders in school setting: learning from a pilot project.

\author{
Ángeles Asiel Rodríguez a , María del Mar García-Gil ${ }^{\text {b }}$, Namdev Freund Llovera ${ }^{\text {c }}$, Alberto Fer- \\ nández Liria ${ }^{d}$.
}

${ }^{a}$ Psiquiatra. Hospital de Día de Adolescentes. Hospital Universitario Príncipe de Asturias, España. ${ }^{b}$ Institut d'Investigació en Atenció Primària (IDIAP Jordi Gol). Facultat de Medicina. Universitat de Girona, España. ${ }^{c}$ Residente de Psiquiatría. Hospital Universitario Príncipe de Asturias, España. ${ }^{d}$ Psiquiatra. Hospital Universitario Príncipe de Asturias. Universidad de Alcalá, España.

Correspondencia: Ángeles Asiel Rodríguez, (basielro@yahoo.es)

Recibido: 22/10/2012; aceptado: 16/02/2013

RESUMEN: Los síntomas prodrómicos del trastorno mental grave debutan en la adolescencia y la juventud. La adolescencia es una etapa clave en el desarrollo biopsicosocial del individuo. La posibilidad de la detección y abordaje precoz del Trastorno Mental Grave (TGM) y la identificación de Estados Mentales de Alto Riesgo (EMAR), podría disminuir el impacto de la enfermedad en el desarrollo psicosocial del adolescente. Una importante limitación en la intervención precoz de adolescentes y jóvenes con TMG es que entre los Institutos de Educación Superior (IES) y los Centros de Salud Mental (CSM) no existe una vía directa, rápida y efectiva de derivación. Nuestro objetivo fue evaluar un programa específico de formación para la detección de EMAR y TMG en 7 Institutos de Educación Superior de Alcalá de Henares. Se utilizaron cuestionarios para evaluar el nivel de conocimientos del personal docente antes y después de realizar talleres formativos diseñados por profesionales de salud mental. Los profesionales de los IES tienen un nivel de conocimiento adecuado sobre la psicosis. La disposición de un sistema de derivación rápido, directo y efectivo entre los IES y los CSM favorece la detección de TMG. A pesar de los conocimientos del profesorado acerca de la psicosis, existen dificultades en la detección de EMAR. No impresiona la tendencia al estigma entre el personal docente, sin embargo el estigma social, familiar e individual dificultan las estrategias de intervención.

PALABRAS CLAVE: psicosis, prevención, institutos de educación superior, trastornos mentales, adolescente.
ABSTRACT: The early symptoms of severe mental disorders appear in adolescence and youth. Adolescence is a critical time for the biopsychosocial development of an individual. The possibility of early detection of mental disorders and the identification of individuals at high risk of psychosis, could lessen the impact of the disease on the psychosocial development of adolescents. A major limitation of early intervention in adolescents and young adults with a severe mental disorder is that there are no direct referral pathways between colleges and Mental Health Network.

Our main goal was to evaluate a specific training program for detection of individuals at high risk of psychosis and severe mental disorders in $7 \mathrm{co}-$ lleges of Alcala de Henares. The questionnaires designed for mental health professionals were used to assess the level of knowledge of teachers before and after training workshops. Higher education teachers have adequate knowledge about psychosis.

The provision of a bypass system, fast and direct, between colleges and community mental health centers facilitates the detection of severe mental disorders. Although teacher knowledge about psychosis, there are difficulties in detecting individuals at high risk of psychosis. The data obtained suggest that teachers have little or no stigma, however, social stigma, family stigma and personal stigma, limited early intervention strategies. KEY WORDS: Adolescent Health, immigrants, alcohol drinking, tobacco, gender identity. 
ORIGINALES Y REVISIONES

\section{Introducción}

A pesar de que no existen datos epidemiológicos precisos, se estima que la prevalencia global de los trastornos mentales en niños y adolescentes en España, es similar a la observada en otros países, y se sitúa entre un 10\%-20\%, según los diferentes estudios (1).

Una reciente revisión sobre la edad de aparición de los trastornos mentales graves, sugiere que los trastornos psicóticos debutan durante la adolescencia y la juventud, lo que se ha relacionado con un aumento de la duración de psicosis sin tratar, y peor pronóstico clínico y funcional de los pacientes (2).

En comparación con la población general, los adolescentes utilizan en menor grado los servicios sanitarios primarios y secundarios (3). Los hallazgos de Potts sugieren que los adolescentes generalmente no consultan por malestar psíquico (4).

Se ha planteado la necesidad de desarrollar estrategias específicas de detección e intervención precoz que aseguren el acceso rápido y efectivo a los recursos (5). Actualmente se están desarrollando múltiples estrategias para fomentar la prevención y la promoción de la salud mental del niño y el adolescente, sin embargo y a pesar de los avances en el tratamiento infanto juvenil existen importantes carencias dentro de los propios dispositivos de salud mental (6). En España se ha reconocido ampliamente esta necesidad en la Estrategia en Salud Mental del Sistema Nacional de Salud (7), y en diferentes Guías de Práctica Clínica del Sistema Nacional de Salud $(8,9)$ comienzan a poner en marcha programas centrados en la atención al trastorno mental grave (TMG) en la infancia y adolescencia (10).

La psicosis precoz incluye la fase de estado mental de alto riesgo (EMAR) o prodrómica, la fase de primer episodio psicótico y la fase de recuperación y período crítico (de 3 a 5 años tras el primer episodio psicótico) (11). Los pródromos de los trastornos psicóticos se caracterizan por la aparición de síntomas inespecíficos y disruptivos. Estudios recientes indican que si los individuos cumplen criterios de "estado mental de riesgo" tienen un 30-40\% de posibilidades de desarrollar psicosis. Entre un 33 y un 58\% aproximadamente de esta población hará una transición a la psicosis (12).

La evolución del trastorno en estas fases de psicosis incipiente y la demora en su tratamiento puede derivar en la denominada "toxicidad psicológica" una alteración en las circunstancias vitales, como fracaso escolar y laboral, autoagresiones, cambios en las relaciones interpersonales y conflictos intrafamiliares, entre otros(13). Es por tanto necesario buscar nuevas fórmulas y realizar un trabajo más activo que permita poder acercarnos a los adolescentes en riesgo de enfermedad mental (14).

En el año 2005 la Organización Mundial de la Salud (OMS) y la Asociación Internacional de Psicosis Temprana (IEPA) proponen una serie de acciones estra- 
ORIGINALES Y REVISIONES

tégicas para la atención precoz en psicosis (15). Numerosos estudios, incluyendo los planes europeos de Helsinki (16) y Bruselas (17), sugieren que las estrategias de intervención precoz contribuyen a disminuir la severidad de la enfermedad, recurrencias y sufrimiento familiar, fomentan la integración, disminuyen el estigma y favorecen la prevención de trastornos psiquiátricos secundarios (2).

Los adolescentes permanecen gran parte de su tiempo dentro de las instituciones educativas, posibilitando un seguimiento longitudinal de su comportamiento, interacción social y desarrollo cognitivo.

\section{Objetivos:}

El propósito inicial de la intervención era evaluar los efectos de un programa específico dirigido a los profesionales de educación que pretendía proporcionarles formación y apoyo para detectar adolescentes con trastorno mental grave o con alto riesgo de desarrollarlo. El contenido fue diseñado por profesionales de salud mental específicamente para ser transmitido a los equipos educativos de los institutos.

Los objetivos específicos de la intervención eran:

1. Evaluar los conocimientos de los profesionales de educación sobre la psicosis, la esquizofrenia, estados mentales de alto riesgo, e identificación precoz de casos.

2. Analizar el efecto de los de talleres formativos en la detección de TMG.

3. Conocer, detectar y tipificar las dificultades de comunicación entre los equipos de orientación y los alumnos y familias al abordar situaciones relacionadas con la enfermedad mental.

4. Consolidar la relación entre salud mental y educación a través del diseño de cauces específicos que faciliten un modelo de trabajo diferente al habitual. Material y Métodos

Selección de centros educativos:

El sector educativo del área Este de la comunidad de Madrid, correspondiente al Área de Gestión Clínica de Psiquiatría y Salud Mental del Hospital Universitario Príncipe de Asturias, esta integrado por 14 Institutos de Educación Superior (IES). Se seleccionaron 7 IES, uno por distrito del municipio. En los distritos en que existía más de un IES, se realizo una selección aleatoria.

Los datos sobre los institutos se solicitaron de la Dirección de Área de la Consejería de educación (Tabla 1). 


\section{Instrumentalización:}

Se elaboró un taller formativo sobre psicosis para sensibilizar al personal docente y de los departamentos de orientación. Los módulos de formación fueron diseñados para identificar los posibles cambios o comportamientos de riesgo de los adolescentes, y de este modo poder realizar un trabajo preventivo posterior. El responsable de impartir el contenido de los talleres fue el coordinador del proyecto. Transcurrieron tres meses entre el primer taller y el final de la intervención (derivación de los pacientes a Salud Mental).

Los responsables de los departamentos de orientación de cada instituto, previo al consentimiento y el apoyo de los responsables del área educativa de la zona Este de la Comunidad de Madrid, se encargaron de seleccionar a los profesionales que participarían en las actividades formativas.

Tabla 1.

\begin{tabular}{|l|c|c|}
\hline \multirow{2}{*}{ Centro } & CURSO 2009/10 & CURSO 2010/11 \\
\cline { 2 - 3 } & ESO/Total & ESO/Total \\
\hline ALKAL'A NAHAR & $497 / 836$ & $479 / 809$ \\
\hline MATEO ALEMÁN & $429 / 807$ & $418 / 920$ \\
\hline ANTONIO MACHADO & $664 / 2198$ & $632 / 2358$ \\
\hline IGNACIO ELLACURÍA & $503 / 753$ & $491 / 738$ \\
\hline DOCTOR MARAÑON & $458 / 701$ & $506 / 726$ \\
\hline ISIDRA DE GUZMAN & $506 / 628$ & $529 / 645$ \\
\hline ATENEA & $265 / 434$ & $287 / 415$ \\
\hline
\end{tabular}

La valoración de las derivaciones al Centro de Salud Mental (CSM), así como la evaluación de los casos estuvo a cargo del coordinador del proyecto, quien según las características del caso, era el encargado de decidir y gestionar la atención preferente en el CSM. Este sistema de derivación suprime la obligatoriedad de las derivaciones desde los centros de atención primaria como medio de acceso a la atención especializada en salud mental, favoreciendo el contacto directo entre los orientadores del Institutos de Enseñanza Superior (IES) y Salud Mental. Se utilizó un formulario de derivación, diseñado por el Servicio de Salud Mental, con los datos personales y clínicos del paciente a evaluar.

\section{Metodología docente:}

Antes de iniciar la primera sesión se solicitó a los participantes que respondieran a un cuestionario (Anexo I) que costaba de dos partes. En la primera parte se recogió información sobre las características generales de los asistentes y en la segunda sobre los conocimientos y aptitudes ante la psicosis. Al no encontrar escalas validadas al español para evaluar los conocimientos de los profesionales de la educación, se diseño un material basado en el cuestionario "Knowledge and 
ORIGINALES Y REVISIONES

Experience of Social Emotional Difficulties among Young people (KESEDY)" utilizado en estudios similares al nuestro $(18,19)$. El taller estaba estructurado en dos sesiones de una hora de duración. Entre cada sesión transcurrieron tres semanas. Para la realización del taller se utilizaron diversos formatos de presentación audiovisual (video, exposición, lecturas de textos y casos prácticos).

Al final de la intervención se pasó un cuestionario de calidad para evaluar las características, metodología, organización y capacidades del equipo docente del taller (Anexo 2).

\section{Programación del contenido teórico}

En la primera sesión se imparten conocimientos elementales sobre psicosis y esquizofrenia, así como una introducción sobre los estados mentales de alto riesgo para la identificación de alumnos con posibles síntomas psicóticos o de alto riesgo, finalizando con un turno de preguntas y debate.

En la siguiente, se comenzaba con un breve resumen de la sesión anterior y posteriormente se discutían las estrategias para el abordaje del alumno en riesgo y aproximación a la familia. Al finalizar la sesión se expusieron las dudas y se discutieron posibles situaciones clínicas.

\section{Instrumentos y variables del estudio}

En el cuestionario pasado al inicio del taller se recogieron los siguientes datos:

- Materia en que desempeñan su actividad. Se decidió dividir el área de conocimiento en 4 grupos: ámbito lingüístico, ámbito científico- tecnológico, ámbito psicosocial y otras materias (música, dibujo educación física).

- Años de docencia.

- Tipo de plaza.

- Pertenencia o no al departamento de orientación.

- El conocimiento sobre la psicosis (Anexo I).

- Modos de actuación ante la psicosis

- Actitud ante el estigma.

Al final del taller se evaluó la satisfacción con la intervención mediante una escala de Likert del 1 al 10 (Anexo 2).

Los resultados de la intervención se miden en términos de:

- Casos de EMAR que nunca han estado en contacto con el dispositivo y se detectan como consecuencia de la intervención en los meses siguientes.

- Casos de EMAR detectados que llegan a ponerse en contacto con los servicios de salud mental.

- Falsos positivos en la derivación de EMAR. 
- Tiempo de espera de los EMAR para ser atendidos por un especialista.

- Casos de EMAR que entran en contacto y que el cabo de 12 meses continuan en contacto o han sido dados de alta por decisión de su facultativo responsable.

- Casos de psicosis o TMG que nunca han estado en contacto con el dispositivo y se detectan como consecuencia de la intervención.

- Casos de TMG detectados que llegan a ponerse en contacto con los servicios de salud mental.

- Falsos positivos en la derivación de TMG.

- Tiempo de espera de los TMG para ser atendidos por un especialista.

- Casos de TMG que entran en contacto que el cabo de 12 meses continua en contacto o han sido dados de alta por decisión de su facultativo responsable.

Los profesionales de salud mental, que impartieron el taller, recogieron sistemáticamente las dificultades en la detección, el manejo, la derivación de los casos identificados o las dificultades en la coordinación con el servicio de salud mental, señaladas por los docentes de los IES durante el curso o las actividades de role playing.

\section{Resultados}

Características de los participantes

Las características de los participantes se especifican en la Tabla 2.

Conocimientos y actitudes sobre la psicosis (Tabla 3)

Esta tabla presenta los resultados obtenidos en los cuestionarios de conocimiento. Cabe destacar que en la primera sesión respondieron el 100\% de los participantes, pero en la segunda solo respondieron 22 de 62 participantes (35\%).

El reconocimiento de la psicosis a partir de las viñetas alcanzaba en la primera evaluación el $87,1 \%$.

Los modos de reacción que se consideraban adecuados y posibles con el sistema actual de derivación (contactar a un familiar) también tuvieron puntuaciones del $85 \%$. La necesidad de tratamiento farmacológico y psicológico era mucho menos reconocida en la primera evaluación ( 29 y $56 \%$ respectivamente) y se modificó tras la intervención (Hasta el 45 y 72,7 \% respectivamente)

En los ítems de estigma las puntuaciones estaban ya en la primera evaluación siempre por debajo del $13 \%$.

Evaluación del curso

La puntuación global media de la evaluación del curso fue de 6,98 puntos 
Tabla 2 .

Características de los participantes.

\begin{tabular}{|l|c|}
\hline & Participantes (n= 62) \\
\hline Materia docente & $27,4(17)$ \\
\hline Lingǘstica & $33,9(21)$ \\
Científica & $21,0(13)$ \\
Psicosocial & $17,7(11)$ \\
Otros & $82,3(51)$ \\
\hline Plaza & $16,1(10)$ \\
Fija & $1,6(1)$ \\
Interinidad & $66,1(41)$ \\
Suplencia & \\
\hline Departamento Orientación & \\
\hline Años Docencia & $25,8(16)$ \\
$\leq 10$ & $40,3(25)$ \\
$11-20$ & $33,9(21)$ \\
$>20$ & \\
\hline
\end{tabular}

Porcentajes (número de casos).

aunque se pudieron observar diferencias entre institutos tal y como muestra la Figura 1 .

Aspectos destacados en la observación realizada por los responsables de salud mental que realizaron el curso respecto a los docentes participantes:

1. Respecto a los conocimientos, habilidades y actitudes y las dificultades para la detección de casos:

a. Se confirmó el grado adecuado de conocimientos y actitudes ante el trastorno mental grave.

b. Entre las dificultades para poder hacer detecciones y derivaciones adecuadas se señalan:

i. Los cambios frecuentes del personal que no permiten tener el tiempo suficiente para sentirse seguro en la derivación.

ii. La inseguridad de los profesionales de educación para abordar la problemática con los familiares.

2. Respecto a la valoración de la relación entre salud mental y los IES, las dificultades que se señalaron fueron:

a. Escaso contacto con los profesionales de salud mental.

b. Falta de cauces regulares para establecer contacto con salud mental

c. Escaso feed back a educación por parte de los profesionales sanitarios una vez iniciado el trabajo en salud mental. 
Tabla 3.

Conocimientos y aptitudes ante la psicosis.

\begin{tabular}{|c|c|c|}
\hline & $\begin{array}{c}1^{\circ}(\mathrm{n}=62) \\
\%(\mathrm{n})^{*}\end{array}$ & $\begin{array}{c}2^{\circ}(\mathrm{n}=22) \\
\%(\mathrm{n})^{*}\end{array}$ \\
\hline $\begin{array}{l}\text { Reconocimiento de la psicosis respecto a otras enfermedades mentales } \\
\text { (Viñetas clínicas) (\% de respuestas correctas) }\end{array}$ & - & \\
\hline - Cuadro depresivo & $75,8(47)$ & $86,4(19)$ \\
\hline - Cuadro psicótico & $87,1(54)$ & $90,4(20)$ \\
\hline - Trastorno de hiperactividad & $82,3(51)$ & $86,4(19)$ \\
\hline \multicolumn{3}{|l|}{ Causas de psicosis (\% de la respuesta más relevante) } \\
\hline - Problema cerebral & $22,6(14)$ & $18,2(4)$ \\
\hline - Abuso de drogas & $17,7(11)$ & $13,6(3)$ \\
\hline - Vulnerabilidad genética & $22,6(14)$ & $63,6(14)$ \\
\hline - Problemas de personalidad & $30,6(19)$ & $9,1(2)$ \\
\hline \multicolumn{3}{|l|}{$\begin{array}{l}\text { Modo de reacción ante la enfermedad (\% de respuesta } 1 \text { que } \\
\text { corresponde siempre) }\end{array}$} \\
\hline - Enviar al médico de cabecera o pediatra & $40,3(25)$ & $31,8(7)$ \\
\hline - Aconsejar un periodo de descanso o vacacional & $1,6(1)$ & $0(0)$ \\
\hline - Enviar a psiquiatría & $38,7(24)$ & $18,2(4)$ \\
\hline - Contactar con un familiar & $85(53)$ & $90,9(20)$ \\
\hline - Hacer una reunión de profesores y tomar una decisión conjunta & $58,1(35)$ & $40,9(9)$ \\
\hline - Tratamiento psicológico & $56,5(35)$ & $72,7(16)$ \\
\hline - Dar tiempo & $3,2(2)$ & $4,5(1)$ \\
\hline - Ingreso en hospital psiquiátrico & $1,6(1)$ & $0(0)$ \\
\hline - Tratamiento farmacológico & $29(18)$ & $45,5(10)$ \\
\hline \multicolumn{3}{|l|}{ Estigma } \\
\hline - Los psicóticos son personas con las que es muy difícil hablar & $12,9(8)$ & $18,2(4)$ \\
\hline - Los psicóticos son personas violentas & $12,9(8)$ & $9,1(2)$ \\
\hline - Los psicóticos no pueden vivir solos & $9,7(6)$ & $22,7(5)$ \\
\hline - Los psicóticos presentan problemas con la justicia con frecuencia & $12,9(8)$ & $4,5(1)$ \\
\hline
\end{tabular}

* Porcentajes (número de casos)

Figura 1.

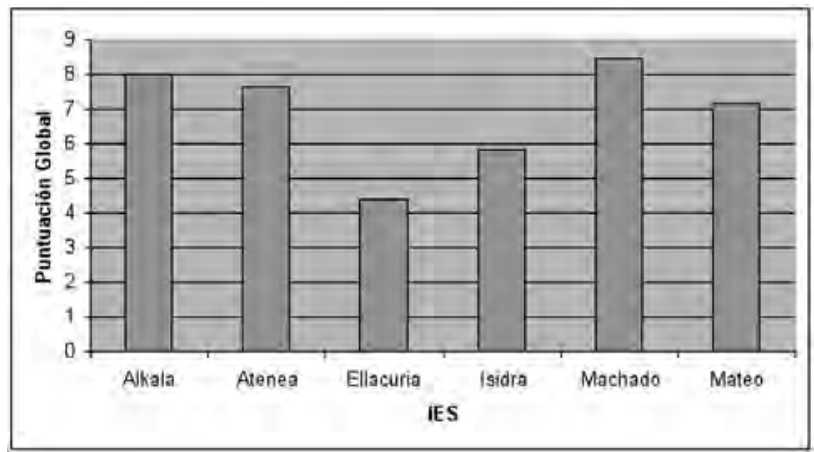




\section{Detección de pacientes}

Como consecuencia de la intervención y el establecimiento de un sistema de derivación asociado a ella, entre marzo y junio de 2010, los profesionales de los IES derivaron a 8 alumnos que anteriormente no habían estado en contacto con el sistema de atención a la salud mental.

Todos los casos detectados presentaban trastornos mentales graves: Siete de ellos recibieron una cita preferentes en el CSM en los siguientes 10 días y uno fue derivado a través del Medico de Atención Primaria (MAP) (se ofreció la posibilidad de derivación directa pero la familia prefirió realizarlo a través del medico de atención primaria). La patología detectada fue la siguiente: 3 casos de psicosis franca, 2 casos de Trastorno generalizado del desarrollo (TGD) 1 caso de Trastorno depresivo mayor (TDM), 1 caso de Trastorno Obsesivo Compulsivo (TOC).

Los pacientes remitidos a través de esta nueva modalidad de derivación fueron atendidos en los servicios especializados en un plazo inferior a 10 días.

El paciente que pidió cita a través del médico de atención primaria no fue evaluado ya que no acudió a la cita.

En el seguimiento realizado 24 meses después de la intervención, la situación de estos pacientes era la siguiente:

- Los pacientes diagnosticados de psicosis han continuado en seguimiento dos años después de la intervención, precisaron tratamiento farmacológico y la utilización de otros recursos (ingreso hospitalario, centro de rehabilitación psicosocial y/o hospital de día de adolescentes).

- De los pacientes con diagnóstico de TGD

o Uno continua en seguimiento y ha sido incluido en un grupo que se realiza en CSM.

o El otro se citó en 3 ocasiones diferentes ya que no acudió e incluso ante las reticencias de la familia se hizo un acompañamiento por parte del centro escolar que permitió una primera evaluación pero la familia no acepto el seguimiento.

- El paciente con TDM continúo en tratamiento durante 18 meses. No continua seguimiento en nuestra área sanitaria por cambio de residencia. La guardia y custodia fue asumida por la oficina del menor de la Comunidad de Madrid

- El paciente con TOC acudió a dos citas y abandono el tratamiento. Cabe destacar que dadas las altas capacidades del paciente, la familia no consideró necesario el seguimiento.

- No se derivaron alumnos con estados mentales de alto riesgo que aún no hubieran desarrollado un trastorno psicótico u otro trastorno mental grave franco.

- No se han detectado falsos positivos de TMG ni de EMAR. 
ORIGINALES Y REVISIONES

\section{Discusión}

Antes de iniciar la intervención, los orientadores y los docentes de los IES seleccionados para este trabajo poseían un nivel de conocimiento adecuado sobre el trastorno mental grave, como se comprobó en los cuestionarios realizados previos al taller. Sus respuestas en el cuestionario tampoco reflejan una actitud altamente estigmatizante hacia los trastornos psicóticos y los planteamientos sobre el abordaje son congruentes a su nivel de conocimientos. La discusión del contenido teórico con el personal docente, ratifica que los profesionales tienen los conocimientos necesarios para identificar posibles casos de enfermedad mental conocen los cauces para un abordaje correcto y no tienen una actitud muy estigmatizadora.

Las derivaciones fueron realizadas en consideración de un criterio de gravedad y repercusión sobre el funcionamiento global del alumno. Los adolescentes que utilizaron los cauces habilitados por el programa fueron atendidos según los plazos acordados, ofreciendo una vía eficaz de aproximación diagnostica y atención precoz del trastorno mental grave en niños y adolescentes. En los casos diagnosticados de psicosis fue necesario realizar intervenciones farmacológicas y psicosociales y su derivación a dispositivos de segunda línea (hospital de día, centro de rehabilitación psicosocial o ingreso hospitalario) debido a la gravedad de la clínica.

Llama la atención, sin embargo, que estos conocimientos y actitudes preexistentes no hayan podido evitar que un número muy elevado de casos con patología grave no hubieran sido puestos en contacto con los servicios de salud mental antes del taller y que no se haya derivado ningún caso de estado mental de alto riesgo ni siquiera después del mismo.

Probablemente la escasa derivación no tiene que ver con la falta de conocimientos o actitudes de los educadores, sino con otros factores, a los que deberían dirigirse los programas de atención temprana, y que fueron señalados en el trabajo de los talleres, como son:

- La desconexión entre el sistema educativo y el sanitario.

- La resistencia asociada al estigma de la familia y los propios alumnos y la dificultad de manejo por parte de los educadores.

- La ausencia de pruebas diagnosticas que avalen la sospecha diagnostica de los profesionales del sector educativo y el temor de etiquetar al alumno con un diagnostico equivocado y con repercusiones psicosociales.

- La falta de confianza sobre los conocimientos de los educadores sobre la detección de casos.

- El cuestionamiento, por parte de los profesionales de salud mental, de los síntomas de alarma detectados por los educadores. 
ORIGINALES Y REVISIONES

Las actividades de contenido más práctico, que tenían lugar al finalizar las sesiones de los talleres, ofrecieron un espacio para el abordaje de dudas clínicas e identificación de dificultades en las entrevistas con familias y alumnos. Es probable que la presencia de un interlocutor cercano, y único intermediario entre la sospecha de trastorno grave y la derivación a salud mental facilito la detección y el inicio del tratamiento.

En algunos de los casos derivados (con diagnósticos diferentes al de psicosis) el tratamiento que hubiera estado indicado en los servicios especializados de salud mental no ha podido realizarse debido a la falta de colaboración de la familia.

Estos hallazgos no son enteramente concordantes con lo reseñado en la literatura sobre motivos de la demora en la detección. En nuestro trabajo falta de reconocimiento de los síntomas prodrómicos (20) parece tener menor importancia que la poca accesibilidad a los equipos de tratamiento (21).

La clave de la detección precoz de los trastornos psicóticos y de la intervención temprana consiste en mantener un elevado índice de alerta entre los grupos con un riesgo mayor (22).

La intervención de los profesionales de los institutos es fundamental para facilitar el acceso de los adolescentes a los servicios de salud mental. Los profesores cumplen un rol esencial en la identificación de cambios precoces en el funcionamiento social y conducta de los adolescente y en la derivación de individuos en riesgo o con síntomas francos de psicosis (22). Los profesores tienen el potencial para la identificación precoz de la psicosis, sin embargo sigue existiendo desconocimiento sobre que hacer y donde acudir cuando un alumno presenta síntomas psicóticos (18). Existe la necesidad de formar a los profesionales de los institutos educativos en los programas de atención primaria y promoción de la salud para favorecer el reconocimiento los síntomas psicóticos y la rápida derivación de los alumnos a los servicios de salud mental $(18,23)$.

El contacto entre un programa especializado en intervención precoz y los profesionales de los institutos, sensibiliza a los profesionales sobre la detección, diagnostico, curso y pronostico de los trastornos psicóticos adecuadamente tratados, lo que pudiera facilitar derivaciones tempranas de adolescentes con signos precoces de psicosis (22).

\section{Dificultades y limitaciones.}

Al no disponer de material validado al español para la evaluar los conocimientos de los profesores dificulta la generalización de los resultados.

El periodo de derivación solo duro 3 meses, debido a dificultades administrativas de los centros en organizar el segundo taller. Hubiera sido interesante que 
el periodo hubiera sido más largo para poder ver como continuaba el número de derivaciones.

La menor participación en el segundo taller pudo estar relacionada con el prolongado intervalo de tiempo entre un taller y otro. Resulta interesante que en los institutos donde la intervención fue más efectiva, la participación fue más estable entre ambos talleres.

Las actividades específicas de formación y apoyo fueron impartidas por la misma persona y siguiendo la misma estructura. Las derivaciones solo se realizaron desde tres IES, en las que se registraron mayores puntuaciones en el cuestionario de satisfacción. Desde nuestro punto de vista, algunos factores que han podido contribuir a estas diferencias son:

- Diferencias entre los perfiles de los asistentes. Cada centro escolar eligió a los asistentes al taller, en unos centros asistieron preferentemente miembros de los equipos de orientación. En estos grupos existía una mayor sensibilidad a las problemáticas planteadas, en otros, sin embargo, se dio la opción que asistiera cualquier tutor.

- Aunque la asistencia fue voluntaria en algunos centros se utilizó espacios de reunión ya establecidos, lo que probablemente facilitara la tarea y en otros el taller se realizó en horas fuera de la actividad docente.

- Otros factores a tener en cuenta pero difícilmente cuantificables son las relaciones que habían existido previamente entre el centro escolar y salud mental o la preocupación, en el momento de la realización de los talleres, por alguno alumno concreto que no estaba siendo atendido desde el punto de vista del equipo docente, del modo esperado por salud mental.

Los resultados del estudio sobre Trastornos mentales graves en la infancia y adolescencia en una población de Molina Segura (24), sugieren que el tiempo medio de espera para evaluación está en torno a los 3,22 meses y teniendo en cuenta que solo el $17 \%$ de las derivaciones proceden a petición del centro escolar podemos considerar que este tipo de intervención pueden se una vía eficaz para acercarnos al diagnostico y a la atención de pacientes con TMG en la infancia y adolescencia. Conclusiones del estudio:

1. En los centros escolares se observo un conocimiento adecuado sobre el TMG y la psicosis, sin tendencia a la estigmatización. Partiendo del nivel basal de conocimiento, no se objetivaron cambios significativo tras la realización del taller, lo que suponemos obedece a un efecto techo. A pasar de esto, al finalizar la intervención, el personal docente parece haber adquirido la capacidad para afinar sobre algunos detalles significativos sobre psicosis (causas de la enfermedad; tratamientos).

2. Tras la intervención, 8 trastornos mentales graves fueron correctamente detectados, derivados y atendidos en un plazo inferior a 10 días.

3. Los trastornos mentales graves detectados no fueron sólo trastornos del es- 
ORIGINALES Y REVISIONES

pectro psicótico (psicosis franca 37,5, Trastorno generalizado del desarrollo 25\%, Trastorno depresivo mayor 12,5, Trastorno Obsesivo Compulsivo 12,5, otros 12,5).

4. Los educadores que asistieron al programa no fueron capaces de detectar EMAR ni antes ni después de realizar la formación.

5. Las principales dificultades para el manejo de alumnos con EMAR o TMG señaladas por los educadores fueron la inseguridad a la hora de confrontar a la familia con las manifestaciones clínicas detectadas en el alumno,

6. La alta rotación de profesionales por los puestos de trabajo dificulta la continuidad del seguimiento y la coherencia de la intervención.

7. Los principales obstáculos señalados por los educadores para la coordinación y colaboración entre los servicios especializados en la atención a la salud mental fueron la ausencia de causes regulares y el escaso contacto con los profesionales de salud mental, así como el escaso feed back aportado por los profesionales sanitarios a los docentes del sector educativo.

8. Una dificultad añadida, es la ausencia de estrategias sistemáticas que aseguren la continuidad del seguimiento de pacientes con EMAR o TMG una vez que han contactado con los Centros de Salud Mental.

\section{Enseñanzas para el diseño de estrategias de intervención en el medio escolar}

- Es posible que en el medio escolar existan trastornos mentales muy graves sin detectar o sin contacto con el sistema sanitario. La actuación sobre este problema puede ser necesaria antes de poder actuar sobre los EMAR

- Es posible que el principal obstáculo para la detección y derivación de trastornos metales no sean los conocimientos, habilidades y actitudes de los profesores, que hemos visto a lo largo del estudio, son adecuados. No parece que la sensibilización sobre la enfermedad mental grave sea la prioridad a la hora de confeccionar un programa sobre detección precoz. La necesidad principal, expresada por los profesores y sobre los equipos de orientación, es conseguir una presencia más activa de salud mental en los IES que permita ofrecer el apoyo a las carencias que expresan tener los profesores respecto a la comunicación con la familia y a la contención si se toma la iniciativa de plantear un diagnostico grave.

- El diseño de programas de detección precoz de TMG deberían superar algunas dificultades:

1. En nuestro país esta dispuesta una estructura, rígida y obligatoria, de derivación a los servicios de atención especializada a través de los centros de atención primaria de salud. Se podría establecer una vía directa, ágil y supervisada, que sustituya el sistema tradicional de derivación. 
2. La falta de contacto entre los profesionales de salud mental y los de educación dificulta la colaboración en estos casos e impide que los segundos reciban el apoyo que necesitarían para sentirse seguros en el manejo de los casos detectados y sus familias. Sería necesario desarrollar estrategias para romper esta brecha.

3. Aunque los educadores no se sitúen en actitudes muy estigmatizantes, el estigma con el que pueden contemplar los trastornos mentales y los modos de tratarlos los propios adolescentes, sus familias y la población general es visto por los educadores como importante y para enfrentarlo, podrían necesitar de instrumentos específicos que habría que desarrollar conjuntamente.

4. Cualquier medida orientada a mejorar la detección deberá completarse con las encaminadas a mejorar la tasa de retención de los servicios especializados de salud mental, lo que supone probablemente incrementar la asertividad de los mismos con este tipo de problemas y diseñar estrategias para trabajar con las actitudes de las familias. 


\section{Anexo 1}

\section{1- RELACIONA LAS VIÑETAS CLÍNICAS (A-B-C) CON LOS DIAGNOSTICOS (1-2-3)}

A.- Varón de 16 años escolarizado que presenta el siguiente cuadro clínico, desconfianza, cree que hay un grupo de chicos de su barrio que le miran raro cuando sale, incluso refiere que ha oído que le insultan. Se muestra muy reticente a salir comenzando a no acudir al IES, pasando gran parte del día en su habitación e irritable con sus padres cuando intentan averiguar que ocurre. B.- Varón de 15 años, escolarizado que presenta la siguiente sintomatología: gran irritabilidad, disminución del rendimiento escolar, apenas realiza tareas, se queja de dormir muy mal por la noche y con menos ganas de comer.

C.- Varón de 15 años que presenta tics faciales (caracterizados por guiños de ojo), muchas dificultades para permanecer en su sitio cuando esta el aula, en ocasiones desafiante con los profesores y adultos. Cuando se habla de estos problemas se muestra muy angustiado y llora con facilidad.

\begin{tabular}{|l|l|}
\hline 1) Cuadro depresivo & \\
\hline 2) Cuadro psicótico & \\
\hline 3) Trastorno de hiperactividad & \\
\hline
\end{tabular}

\section{2- CUAL DE LOS SIGUIENTES FACTORES SON CAUSA DE PSICOSIS}

(Puntúa entre 1-4 lo que parezca más relevante, siendo el 1 lo más relevante)

\begin{tabular}{|l|l|}
\hline Problema cerebral & \\
\hline Abuso de drogas & \\
\hline Vulnerabilidad genética & \\
\hline Problemas de personalidad & \\
\hline
\end{tabular}

3- PUNTUA ENTRE 1- 5 LO QUE HARÍA SI SE ENCONTRARA CON UN ALUMNO PSICOTICO (1. Siempre, 2. con mucha frecuencia, 3. algunas veces 4. esporádicamente, 5. Nunca)

\begin{tabular}{|l|l|}
\hline Enviar al médico de cabecera o pediatra & \\
\hline Aconsejar un periodo de descanso o vacacional & \\
\hline Enviar a psiquiatría & \\
\hline Contactar con un familiar & \\
\hline Hacer una reunión de profesores y tomar una decisión conjunta & \\
\hline
\end{tabular}

4- TIPOS DE TRATAMIENTO: PUNTUE ENTRE 1 -5 LOS TRATAMIENTOS EN FUNCIÓN DE LA EFICACIA SEGÚN SU OPINION ( 1.siempre, 2. con mucha frecuencia, 3. algunas veces, 4. esporádico, 5. nunca)

\begin{tabular}{|l|l|}
\hline Tratamiento psicológico & \\
\hline Dar tiempo & \\
\hline Ingreso en hospital psiquiátrico & \\
\hline Tratamiento farmacológico & \\
\hline
\end{tabular}

5- QUÉ OPINA SOBRE LAS SIGUIENTES ASEVERACIONES (puntúa del 1 al 5 como en preguntas anteriores)

\begin{tabular}{|l|l|}
\hline Los psicóticos son personas con las que es muy difícil hablar & \\
\hline Los psicóticos son personas violentas & \\
\hline Los psicóticos no pueden vivir solos & \\
\hline Los psicóticos presentan problemas con la justicia con frecuencia & \\
\hline
\end{tabular}




\section{Anexo 2 \\ CUESTIONARIO EVALUACIÓN ACTIVIDADES DE FORMACIÓN \\ CURSO DE TALLER DE DETECCIÓN PRECOZ DE ENFERMEDAD MENTAL GRAVE}

Valore según la siguiente escala, los aspectos que se detallan referidos a la actividad de formación.

\begin{tabular}{|c|c|c|c|}
\hline NADA ADECUADO & POCO ADECUADO & ADECUADO & MUY ADECUADO \\
\hline $\mathbf{1}$ & $\mathbf{2}$ & $\mathbf{3}$ & $\mathbf{4}$ \\
\hline
\end{tabular}

\section{OBJETIVOS DEL CURSO}

\begin{tabular}{|l|l|l|l|l|}
\hline La claridad en la definición de objetivos fue & 1 & 2 & 3 & 4 \\
\hline El grado de cumplimiento de los objetivos del curso fue & 1 & 2 & 3 & 4 \\
\hline
\end{tabular}

\section{CONTENIDOS DEL CURSO}

\begin{tabular}{|l|l|l|l|l|}
\hline El contenido temático en relación a los objetivos del curso ha sido & 1 & 2 & 3 & 4 \\
\hline
\end{tabular}

\section{METODOLOGÍA DEL CURSO}

La calidad de las exposiciones teóricas ha sido

La integración entre las clases prácticas y las exposiciones teóricas han sido

\begin{tabular}{|l|l|l|l|}
\hline 1 & 2 & 3 & 4 \\
\hline 1 & 2 & 3 & 4 \\
\hline
\end{tabular}

\section{ORGANIZACIÓN}

\begin{tabular}{|l|l|l|l|l|}
\hline El cumplimiento del horario previsto ha sido & 1 & 2 & 3 & 4 \\
\hline El horario en que se ha realizado te ha parecido & 1 & 2 & 3 & 4 \\
\hline La presentación de dicho material ha sido de una calidad & 1 & 2 & 3 & 4 \\
\hline
\end{tabular}

\section{DOCENTE}

\begin{tabular}{|l|l|l|l|l|}
\hline La motivación conseguida ha sido & 1 & 2 & 3 & 4 \\
\hline
\end{tabular}

6. RESALTA LO QUE MÁS TE HA GUSTADO DEL CURSO

7. RESALTA LO QUE MENOS TE HA GUSTADO DEL CURSO

8. LA VALORACIÓN GLOBAL QUE TE HA MERECIDO EL CURSO HA SIDO (SEÑALA CON UNA "X")

(ESCALA DESDE 1 DEFICIENTE A 10 EXCELENTE)

\begin{tabular}{|l|l|l|l|l|l|l|l|l|l|}
\hline 1 & 2 & 3 & 4 & 5 & 6 & 7 & 8 & 9 & 10 \\
\hline
\end{tabular}

\section{COMENTARIOS Y SUGERENCIAS}


ORIGINALES Y REVISIONES

\section{BIBLIOGRAFÍA:}

(1) Arango C, Franco C.Prevalencia de trastornos mentales en niños y adolescentes. Monografías de Psiquiatría [Internet]. 2004;16:19-27.

(2) de Girolamo G, Dagani J, Purcell R, Cocchi A, McGorry PD. Age of onset of mental disorders and use of mental health services: needs, opportunities and obstacles. Epidemiology and psychiatric sciences. 2012; 21(1): 47-57. Epub 2012/06/08.

(3) Grifo M, Ramos M, Conde J, Gil L, Izquierdo J, Martínez O. Problemas de salud de adolescentes en un centro de salud urbano. Semergen: Revista Española de Medicina de Familia. 1997;11:694-6.

(4) Potts Y, Gillies ML, Wood SF. Lack of mental well-being in 15-year-olds: an undisclosed iceberg? Family practice. 2001;18(1):95-100. Epub 2001/01/06.

(5) Asociación_Española_de_Neuropsiquiatría. Consenso sobre Atención Temprana a la Psicosis. Madrid 2009.

(6) Asociación_Española_de_Neuropsiquiatría. Informe sobre la salud mental de niños y adolescentes. Madrid2009.

(7) Ministerio_de_Sanidad_y_Consumo. Estrategia en Salud Mental del Sistema Nacional de Salud. Madrid2006.

(8) Ministerio_de_Sanida_y_Politica_Social.Guía de Práctica Clínica sobre la Esquizofrenia y el Trastorno Psicótico Incipiente2009.

(9) Ministerio_de_Sanida_y_Politica_Social. Guía de Práctica Clínica sobre la Depresión Mayor en la Infancia y en la Adolescencia. Galicia2009.

(10) Consell_Assessor_d'Assistència_Psiquiàtrica_i_Salut_Mental_a_Catalunya_Servicio_ Catalán_de_la_Salud. Recomendaciones para la atención de los trastornos mentales graves en la infancia y la adolescencia. Barcelona 2003.

(11) Birchwood M, Todd P, Jackson C. Early intervention in psychosis. The critical period hypothesis. The British journal of psychiatry Supplement. 1998;172(33):53-9. Epub 1998/10/09.

(12) Larsen TK, Friis S, Haahr U, Joa I, Johannessen JO, Melle I, et al. Early detection and intervention in first-episode schizophrenia: a critical review. Acta Psychiatr Scand. 2001;103(5):323-34. Epub 2001/05/31.

(13) McGorry PD, Yung AR, Phillips LJ, Yuen HP, Francey S, Cosgrave EM, et al. Randomized controlled trial of interventions designed to reduce the risk of progression to first-episode psychosis in a clinical sample with subthreshold symptoms. Archives of general psychiatry. 2002;59(10):921-8. Epub 2002/10/09.

(14) Tizón J. Bases para un equipo de atención precoz a los pacientes con psicosis. Rev Asoc Esp Neuropsiq. 2009;XXIX(103):35-62.

(15) Bertolote J, McGorry P. Early intervention and recovery for young people with early psychosis: consensus statement. The British journal of psychiatry Supplement. 2005;48:s116-9. Epub 2005/08/02.

(16) World_Health_Organization. Mental Health: Facing the Challenges, Building Solutions : Report from the WHO Regional Office for Europe; 2005.

(17) European_Commission. Improving the mental health of the population: Towards a strategy on mental health for the European Union. Brussels: European Commission; 2005. 
(18) Collins A, Holmshaw J. Early detection: a survey of secondary school teachers' knowledge about psychosis. Early intervention in psychiatry. 2008;2(2):90-7. Epub 2008/05/01.

(19) Doherty R, McLoughlin K, Johnson S. The KEYS study: a survey of knowledge about psychosis and views about services of community and educational staff. Schizophr Res. 2006;86:s122.

(20) Singh SP, Grange T. Measuring pathways to care in first-episode psychosis: a systematic review. Schizophr Res. 2006;81(1):75-82. Epub 2005/11/29.

(21) Larsen TK, Melle I, Auestad B, Friis S, Haahr U, Johannessen JO, et al. Early detection of first-episode psychosis: the effect on 1-year outcome. Schizophr Bull. 2006;32(4):758-64. Epub 2006/07/01.

(22) Langeveld J, Joa I, Larsen TK, Rennan JA, Cosmovici E, Johannessen JO. Teachers' awareness for psychotic symptoms in secondary school: the effects of an early detection programme and information campaign. Early intervention in psychiatry. 2011;5(2):115-21. Epub 2011/03/01.

(23) Peterson FL, Cooper RJ, Laird JM. Enhancing teacher health literacy in school health promotion: a vision for the new millennium. The Journal of school health. 2001;71(4):138-44. Epub 2001/05/17.

(24) Rosagro-Escamez F, Robles-Sánchez F. Trastorno mentales graves en la infancia y adolescencia en una población de Molina de Segura. $12^{\circ}$ Congreso Virtual de Psiquiatría [Internet]. 2011. Available from: www.interpsiquis.com. 